\title{
Barrett's esophagus: natural history
}

\author{
Henry D. Appelman, ${ }^{1}$ Asad Umar, ${ }^{2}$ Roy C. Orlando, ${ }^{3}$ Stephen J. Sontag, ${ }^{4}$ Sanjay Nandurkar, ${ }^{5}$ \\ Hala El-Zimaity, ${ }^{6}$ Angel Lanas, ${ }^{7}$ Paolo Parise, ${ }^{8}$ René Lambert, ${ }^{9}$ and Helen M. Shields ${ }^{10}$ \\ ${ }^{1}$ Department of Pathology, University of Michigan, Ann Arbor, Michigan. ${ }^{2}$ Division of Cancer Prevention, National Cancer \\ Institute, Rockville, Maryland. ${ }^{3}$ Department of Cell and Molecular Physiology, University of North Carolina School of Medicine, \\ Chapel Hill, North Carolina. ${ }^{4}$ Veterans Administration Hospital, Hines, Illinois. ${ }^{5}$ Department of Gastroenterology, Box Hill \\ Hospital, Box Hill, VIC, Australia. ${ }^{6}$ University Health Network, Anatomic Pathology, Toronto General Hospital, Toronto, Canada. \\ ${ }^{7}$ Department of Digestive Diseases, University of Zaragoza, Aragón Health Research Institute, CIBERehd, Zaragoza, Spain. \\ ${ }^{8}$ Department of General Surgery IV, Regional Referal Center for Esophageal Pathology, Pisa, Italy. ${ }^{9}$ International Agency for \\ Research on Cancer, Lyon, France. ${ }^{10}$ Gastroenterology Division, Harvard Medical School and Beth Israel Deaconess Medical \\ Center, Boston, Massachusetts
}

The following on the natural history of Barrett's esophagus (BE) includes commentary on histological sequences of the development of Barrett mucosa; the transformation of esophageal cells from squamous to columnar phenotype; the stages of natural history of dysplasia; the difficulties of predicting progression of dysplasia to adenocarcinoma; the preferable biopsy protocols; the role of Helicobacter pylori infection and gastric atrophy in the risk of BE; the value of decrease of proton pump inhibitor efficacy following eradication of $H$. pylori; the place of antireflux surgery in the natural history of BE; the newest procedures for the endoscopic detection of early neoplasia; and the essential importance of a good understanding of the natural history for the best management of high-grade dysplasia.

Keywords: reflux; adenocarcinoma; CDX2; dilated intercellular spaces; bile salts; high-grade dysplasia; Barrett's esophagus; biopsy protocol; adenocarcinoma; gastric atrophy; H. pylori; GERD; endoscopic grading system; Cag A-postive strain; metaplasia; laparoscopic surgery; high resolution endoscopy; narrow band imaging; trimodal technology; bimodal protocol; esophageal metaplasia; radiofrequency ablation; endoscopic mucosal resection; interobserver reproducibility

\section{Concise summaries}

- The natural history of Barrett's esophagus (BE) with high-grade dysplasia (HGD) remains an enigma.

- Some patients have developed adenocarcinoma very rapidly after the diagnosis of the first HGD. Others have waxed and waned for years, with some regressing to lesser grades of dysplasia, some regressing to no dysplasia (ND), and still some others just living happily with continued HGD.

- It can be assumed that every esophageal mucosa in Barrett's patients started as out as normal squamous mucosa that was then severely damaged by reflux of acid, duodenal contents, or both. From a molecular and genetic standpoint, we know a great deal about the changes that occur once Barrett's mucosa has developed during the dysplasia to carcinoma sequence, but we have hardly any information on comparable genetic and molecular changes in the squamous mucosa that turns it into columnar.

- Despite decades of research, current management of esophageal adenocarcinoma (EA) is still inadequate because at-risk patients are rarely identified. However, it now seems possible to develop a clinical risk classification tool that can probably be further refined and improved using molecular characterization including genetic and epigenetic alterations of early disease.

- Since it is present and to full length on the initial endoscopy, it can be inferred that BE phenotype is generated early in the course of disease. It is also a silent lesion even when exposed to high levels of pathologic acidity, which supports the 
view that BE is a form of "adaptive protection" for the defense of the host against ongoing acid damage to the esophageal wall.

- There is good evidence that has shown that mucosal irregularity, nodules, and ulcers with the Barrett's segment are highly associated with the presence of dysplasia and/or cancer. Thus, close scrutiny of the Barrett's segment to identify surface irregularity and getting targeted biopsies from those areas is extremely important before embarking on 4-quadrant biopsies lest those landmarks get obliterated by blood during sampling. Confocal laser endomicroscopy is a revolutionary technique that images subcellular tissue in real time (virtual biopsy). However, it samples only a highly limited surface area, is time consuming, and early reports to date have not shown it to be better than standard biopsy protocol.

- The trimodal endoscopic protocol requires a specialized material restricted to some reference centers, and random biopsies are still recommended in asymptomactic patients with $\mathrm{BE}$ for the detection of neoplasia in Western countries. In Japan, the detection of suspicious areas at the surface of the mucosa is completed by characterization through categories of the pit pattern and vascular pattern, and the best method of detection of flat areas of neoplasia relies on a bimodal HRE-NBI protocol coupled to magnification.
- Epidemiologic data suggest that a subset of Helicobacter pylori-infected patients may experience a lower risk of Barrett's carcinoma and, altogether, studies suggest that gastric atrophy associated with $H$. pylori infection may be responsible for the inverse association with esophageal disease. However, a negative association has been found in patients without atrophic gastritis, and $H$. pylori infection may increase gastric secretion in patients with antral gastritis.

- There are no studies to define whether H. pylori eradication worsens or improves $\mathrm{BE}$ after $H$. pylori eradication, but indirect evidence suggests that a potential decrease of proton pump inhibitor (PPI) efficacy should not preclude the indication for eradication.

- Even though antireflux surgery seems to better promote regression of BE or dysplasia than medical therapy, it has not demonstrably reduced the incidence rate of adenocarcinoma.

- The wise management of HGD is based on understanding that there is variability in the diagnosis of HGD by pathologists. At present, endoscopic mucosal resection therapy with or without radiofrequency ablation appears to be reasonable therapy for HGD or early esophageal carcinoma. Research data also favor the use of radiofrequency ablation compared to photodynamic therapy because of fewer complications and better efficacy.

\section{What is proof that Barrett's mucosa develops in areas of damaged, previously normal squamous mucosa?}

Henry D. Appelman

appelman@umich.edu

Someone must know the answer to this question, so I tried to find out who, and I felt that it was wise to start by asking the experts. To quote Kahrilas and Pandolfino, two acknowledged esophageal experts writing in a major gastroenterology textbook, "while the cause of Barrett's metaplasia is uncertain, it is clearly associated with gastroesophageal reflux disease (GERD) and believed to occur as a consequence of excessive esophageal acid exposure." According to Spechler, Fitzgerald, Prasad, and Wang, four more acknowledged esophageal experts, "long-segment BE is associated with GERD and the epithelial metaplasia characteristic of $\mathrm{BE}$ is widely regarded as a consequence of GERD." ${ }^{\prime 2}$ According to the website of the Society of Thoracic Surgeons, "the exact reasons for the development of $\mathrm{BE}$ are unknown. Most physicians believe that the damage to the squamous mucosa, which leads to the development of $\mathrm{BE}$ is caused by chronic reflux of acid or other stomach contents."3

Therefore, we assume that every esophageal mucosa in Barrett's patients started out as normal squamous mucosa that was then severely damaged by reflux of acid, duodenal contents, both, or something else. The damaged squamous epithelium somehow was prevented from regenerating, and, 
instead, it was replaced by metaplastic columnar epithelium. One problem with this concept is that we pathologists commonly see significant damage to esophageal squamous mucosa in biopsies, including ulcers and erosions, and what we always see is regenerating squamous epithelium rather than replacement of the squamous epithelium by metaplastic columnar epithelium.

From a molecular and genetic standpoint, we know a great deal about the changes that occur once Barrett's mucosa has developed during the dysplasia to carcinoma sequence. ${ }^{2}$ However, we have hardly any information on comparable genetic and molecular changes in the squamous mucosa that turns it into columnar.

There are some studies using in vitro esophageal squamous mucosal tissue or squamous cell cultures that indicate that acid and/or bile induce columnar type changes in these systems. ${ }^{2}$ In one such study, CDX2, an intestinal differentiation factor, was upregulated in cultured squamous cells. In another study, the expression of CDX1, also an intestinal differentiation factor, was induced in cultured squamous cells. In a third study, there was increased BMP4 expression in stromal cells, which led to columnar cell keratin expression in the squamous cells. Then there was a study that indicated that there was upregulation of HB/EGF in lamina propria fibroblasts under damaged squamous epithelium, which promoted CDX2 in the squamous cells.

Thus, there are changes in squamous cells induced by reflux-type substances that might stimulate columnar metaplasia in the squamous mucosa in the laboratory, but we need to know if these factors actually cause this metaplasia in people. We can manipulate dog esophagi and gastro-esophageal junctions to produce columnar mucosa in the distal esophagus in response to acid, but these are animal models, and we don't have a comparable model in people. Therefore, we have some hints of the molecular and genetic determinants that might turn squamous cells into columnar cells in experimental models, but we do not know if these are the same determinants that work in people with Barrett's mucosa. We also have not identified the progenitor cells that lead to columnar metaplasia following damage to squamous epithelium. Presumably these are some type of stem cells, but we don't know that for a fact.
To summarize, Barrett's mucosa does occur. Epidemiologic evidence suggests that reflux of gastric acid and/or duodenal contents is important in its development. These reflux-type substances can produce molecular and genetic alterations in esophageal squamous cells in the laboratory, but we have no direct evidence that these substances cause columnar metaplasia of squamous mucosa in people.

\section{In GERD patients, can the risks factors for development of Barrett's mucosa be determined? Is it possible to envisage a clinical risk classification tool?}

\section{Asad Umar}

asad.umar@nih.gov

While the overall incidence and mortality rate of many cancers have declined in recent years, the incidence of esophageal adenocarcinoma (EA) has continued to rise. EA incidence has increased in the last three decades more than $600 \%$ in men aged 65 years and older and, notwithstanding progress in multimodality therapies, the overall five-year survival rate is still estimated at $15 \% .^{4,5}$ It is estimated that more than 16,000 new cases of EA will be diagnosed in the United States alone in 2010. Furthermore, esophagectomy with or without neoadjuvant radiation therapy often incurs early postoperative complications and devastating long-term functional abnormalities. Likewise, medical therapies are still highly toxic, and remain unsatisfactory in response duration and overall survival benefit. The completion of any therapy for EA is most often followed by tumor recurrence or distant metastasis that leads to severe morbidities and eventual death. The natural course of the disease is insidious and debilitating, becoming clinically apparent only in advanced stages that are refractory to treatment and are resource intensive. Effective cancer preventive measures will benefit not only affected individuals but also the public at large.

Histologically, EA is thought to arise from the injury of the esophageal mucosa by frequent reflux of gastric contents that result in a sequence of metaplasia to low-grade dysplasia (LGD), HGD, and carcinoma. The metaplasia to dysplasia phase of this sequence is known as BE. BE is diagnosed in approximately $10 \%$ of patients who are referred to 
endoscopy for GERD symptoms. ${ }^{6,7} \mathrm{BE}$, the only widely accepted precursor lesion to EA, is defined as the replacement of the normal squamous epithelium of the distal esophagus by columnar epithelium with intestinal metaplasia. Patients with BE are at least 30 times more likely to develop EA than patients without BE.

Currently, chronic GERD patients are screened for $\mathrm{BE}$ and, if diagnosed to have BE, they are subjected to endoscopic surveillance. Studies demonstrate that patients with malignancy detected at surveillance endoscopy have cancers at an earlier stage and better survival than those with no surveillance. The value of surveillance, however, is questioned by other studies demonstrating that only about $60 \%$ of patients diagnosed with EA have a prior diagnosis of GERD and less than $5 \%$ have BE. Furthermore, although the increased risk of EA in BE patients has been observed consistently, only $0.5 \%$ of endoscopically monitored BE patients progress to EA per year (with the HGD patients progressing at an annual rate of 5-20\%). These findings suggest that despite decades of research, current management of EA neoplasia is still inadequate because at-risk patients are rarely identified. Moreover, criteria for screening and endoscopic surveillance still need to be established in a randomized trial. Given the threat of a highly lethal cancer, both patients and physicians typically opt for the most aggressive strategies for treatment and surveillance even with procedure-related risks.

Clinical risk classification tools need to be developed for better management of GERD of patients. Age, ethnicity, tobacco and/or alcohol use, obesity, and family history make for risk factors for GERD. It is estimated that over 60 million report heartburn or acid indigestion at least one time per month in the United States alone. Over 15 million individuals experience heartburn daily and frequent heartburns (two or more times per week) may be associated with GERD. This is significant, as approximately $10 \%$ of GERD patients develop BE, which eventually is a risk factor for esophageal cancer. ${ }^{3,4}$ Gerson et al. used a multiple logistic regression analysis to predict $\mathrm{BE}$ using age, ethnicity, gender, dysphagea, heartburn, nausea, belching, nocturnal pain, odynophagi, and pain relief with food intake. ${ }^{8}$ Therefore, using a scale of 0-550 total points, a score of 397.4 or higher was strongly associated with an increased risk of $\mathrm{BE}$ while 351.3 for lower risk. This demonstrates that it is possible to develop a clinical risk classification tool, and it can probably be further refined and improved using molecular characterization including genetic and epigenetic alterations of early disease.

\section{Can it be stated that BE follows a phenotypic presentation model in the natural history of GERD?}

\author{
Roy C. Orlando \\ roy_orlando@med.unc.edu
}

The question posed in the title can be more succinctly stated as, "does $\mathrm{BE}$ evolve from GERD?" The answer succinctly stated is, "probably, yes!"

The case in support of this conclusion is based on the following observations:

- $\mathrm{BE}$ is more common in GERD $(\sim 10 \%)$ than in the general population, which has been estimated by a Swedish study to be in the range of $1.6 \%{ }^{9}$

- BE is located in the distal esophagus and associated with exposure of the region to pathologic levels of acid and bile reflux.

- BE exhibits a pattern of growth that appears to parallel those of reflux damage to the distal esophageal epithelium.

- BE has been reported to appear in areas of erosive esophagitis that biopsy did not previously show had evidence for its presence.

- BE has been observed to emerge in the proximal esophagus above an esophagogastric anastamosis following esophagectomy for BE. ${ }^{10}$

- BE has been observed to emerge in the esophagus of animal models of reflux-induced damage to native esophageal stratified squamous epithelium. ${ }^{11}$ Further, and consistent with this in vivo data, are the observations in vitro that esophageal squamous cells exposed to acid and/or bile salts can express CDX2, a gene that favors transformation from squamous to the columnar phenotype. ${ }^{12}$

- The esophageal stratified squamous epithelium above BE has been reported to exhibit the lesion of dilated intercellular spaces. ${ }^{13}$ This lesion is notable for being a feature of acid injury to both animal and human esophageal squamous 
epithelium, and a histopathological correlate of the symptom of heartburn in GERD.

- When refluxate acidity is reduced by treatment with PPIs, islands of squamous epithelium reappear within regions of BE; and when reduced by PPI therapy, areas of distal esophagus from which $\mathrm{BE}$ has been ablated are repaired by a (neo)-squamous epithelium that phenotypically resembles the native esophageal squamous epithelium.

The three observations that prevent full acceptance of this concept include the fact the majority of patients with Barrett's have never been diagnosed with GERD before the diagnosis of BE; the fact that a significant number of those with $\mathrm{BE}$ are asymptomatic and have never had a history of GERD; and the fact that on endoscopy and irrespective of the presence or degree of active esophagitis, BE presents at its full length, a length that neither significantly increases nor decreases over time.

In summary, the weight of the evidence is that $\mathrm{BE}$ arises as a consequence of GERD. Since it is present and full length at the initial endoscopy, it can be inferred that BE phenotype is generated early in the course of disease, and, further, because there are large disparities in the frequency of BE among different ethnicities. The appearance of $\mathrm{BE}$ requires an inherent host susceptibility. BE is also a silent lesion even when exposed to high levels of pathologic acidity, which supports the view that $\mathrm{BE}$ is a form of "adaptive protection" for the defense of the host against ongoing acid damage to the esophageal wall.

\section{Current knowledge on the natural history of Barrett's dysplasia regarding the best management of patients with HGD}

\section{Stephen J. Sontag}

sontagsjs@aol.com

The natural history of BE with HGD remains an enigma. Some patients have developed adenocarcinoma very rapidly after the diagnosis of the first HGD. Others have waxed and waned for years, with some regressing to lesser grades of dysplasia, some regressing to ND, and still some others are living happily with continued HGD. We present unpublished data on two flat Barrett's HGD patients with completely different courses during observation periods ranging from 9 to 18 years.

Patient number 1 was 63 years old when he was diagnosed with HGD at the first esophagogastroduodenoscopy (EGD). For the next 15 years, his course was as follows (Fig. 1, patient 1):

- 37 EGDs,

- 1,455 histologic specimens, and

- $43 \%$ of the 1,455 specimens had HGD.

After 15 years of continuous flat HGD, a small nodule developed and AdCa appeared for the first time. For the subsequent two years, Patient number 1 was treated with various ablative procedures. $\mathrm{He}$ is currently doing well at the age of 80 .

Patient number 2 was a 66 -year-old white male when he was diagnosed with HGD at two outside hospitals. In a three-month period, he was diagnosed with EGD by three different endoscopists at two different hospitals. HGD was diagnosed by three different pathologists. The patient selfreferred himself to our hospital. After his initial diagnosis of HGD at the outside hospitals, his course for the next 11 years at our institution was as follows (Fig. 1, patient 2):

- 15 EGDs;

- 259 histologic specimens demonstrating either ND or no intestinal metaplasia; and

- absolutely no specimens demonstrating LGD, HGD, or AdenoCa.

This patient demonstrates the exact opposite of our first patient. After being diagnosed with HGD at three outside clinics (histologic samples confirmed by our pathologist as well as three outside pathologists), the patient never again showed any evidence of dysplasia on 15 EGDs and 259 specimens. As of this date, he is doing great.

\section{Conclusion}

The biologic influences controlling the fate of HGD are not understood. Our two examples (unpublished data) clearly demonstrate that not all patients with HGD have predictable responses, and the best management for one patient may not be the best management for another patient. Research is needed to discover the influencing factors, but, meanwhile, each patient with flat HGD needs to be assessed as an individual patient. 


\section{Patient 1}

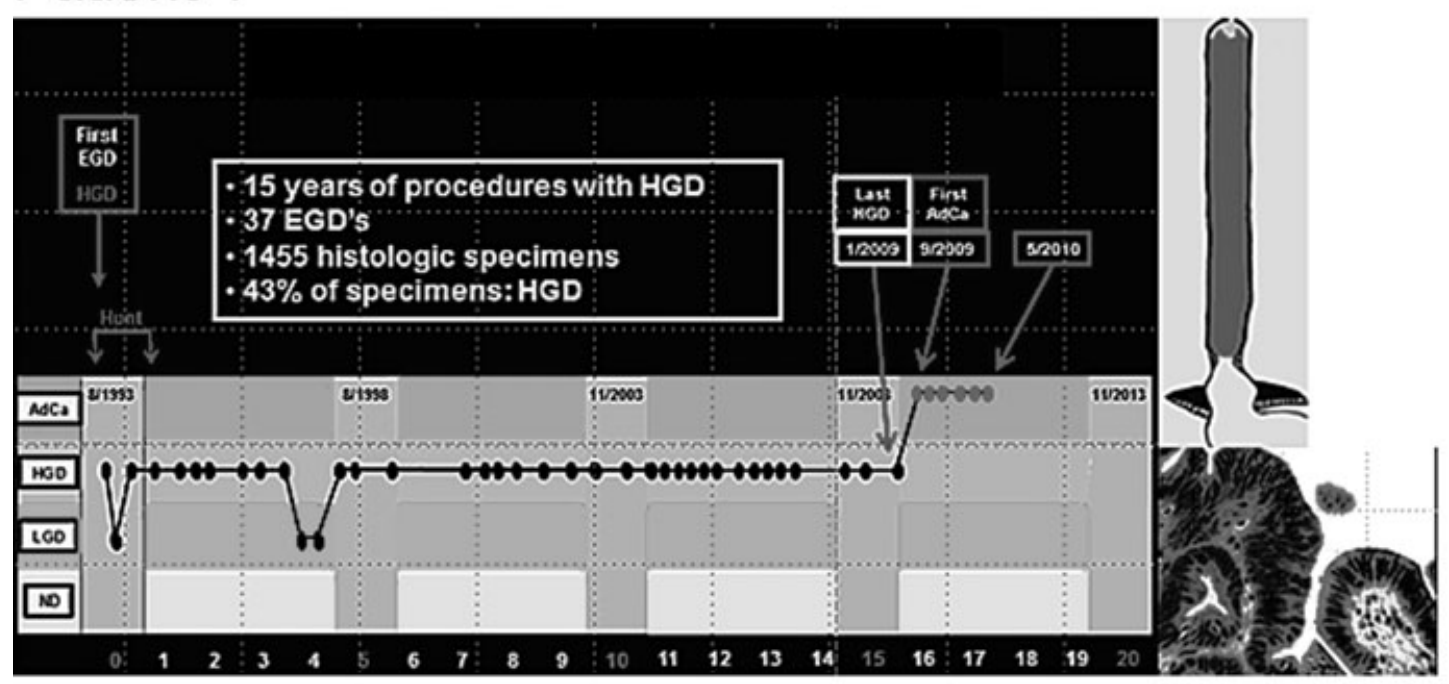

\section{Patient 2}

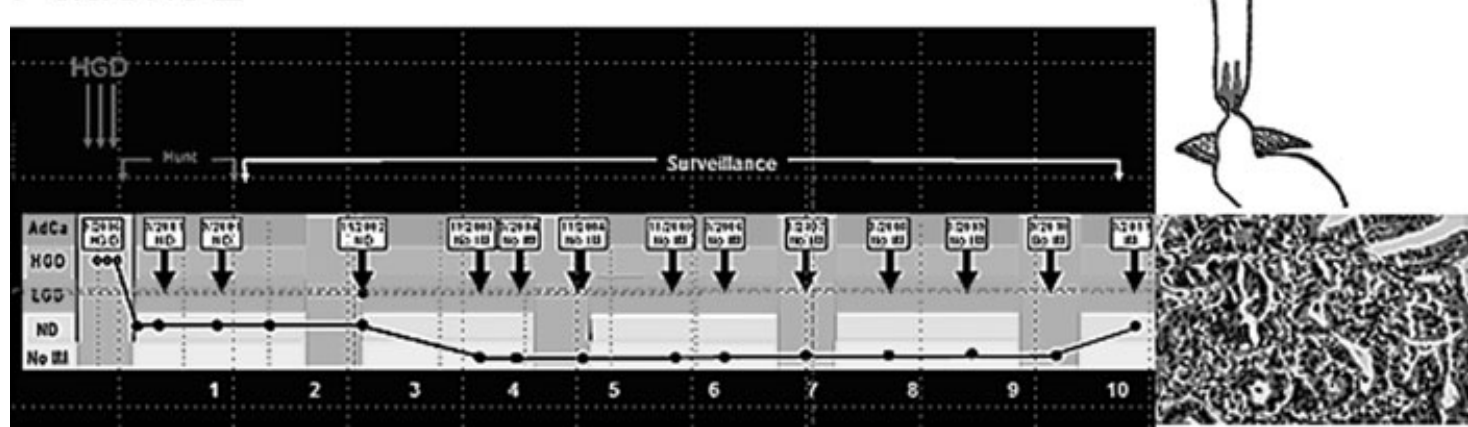

Figure 1.

\section{Is it possible to predict which patients with HGD will progress to adenocarcinoma?}

Stephen J. Sontag

sontagsjs@aol.com

$\mathrm{BE}$ remains a premalignant metaplasia of the esophageal mucosa with a spectrum of cellular behavior ranging from ND to LGD to HGD to cancer (AdCa). Current follow-up recommendations are usually ignored for (flat) HGD: follow-up every three months with EGD and biopsy until HGD disappears or develops into a lump and ablation therapy with any of five modalities.

\section{Objectives}

To offer safe, feasible, and evidence-based $\mathrm{BE}$ surveillance strategies comprising long-term follow-up guidelines with which physicians as well as patients can feel comfortable.

\section{Methods}

Our Veterans Association (VA) outpatient scope room was opened in 1979. Esophagogastroduodenoscopies (EGDs) were performed by one of three endoscopists using preestablished and agreed-upon clinical criteria for (1) EGD indications, (2) definitions, and (3) biopsy technique. Locations, configurations, and extent of $\mathrm{BE}$ and hiatal hernia were recorded and mapped by hand on to one of 22 diagram figures that adjusted for hiatal hernia (HH) size and shape. Attempts were made to biopsy the squamo-columnar junction (SCJ) regardless of its appearance. Biopsy specimens were taken as follows: 
- Two for every $1 \mathrm{~cm} \mathrm{BE}$, or four for every $2 \mathrm{~cm}$ of $\mathrm{BE}$ (one from each quadrant).

- Targeted biopsies were taken of irregular or suspicious areas.

- Biopsy specimens (for all 26 years) were read in detail by one pathologist (GC).

\section{Definitions}

- BE: intestinal metaplasia (IM) from the tubular esophagus or from the GE junction area IF the biopsy contained, as one specimen, the squamoIM junction.

- Prevalent HGD or AdenoCa: diagnosis at the first (or within 12 months of the first) EGD.

- Incident HGD or AdenoCa: diagnosis at least one year after the first EGD.

- Surveillance: time period beginning one year after the first EGD in patients with no current or past HGD or AdenoCa.

\section{Protocol}

Patients were categorized according to the most advanced lesion (e.g., HGD and AdCa occurring together were considered AdenoCa) and remained in the category until a more advanced lesion was found.

- For LGD: biopsy repeated once at one to two years and then every two to four years;

- For HGD: biopsy repeated at three-month intervals for one year ("the hunt");

- If no HGD was found on two consecutive biopsies, intervals became six months;

- After another year, intervals became 12 months, until and unless HGD was again noted.

\section{Results (unpublished data)}

During the 26 years, 1,664 patients were found with $\mathrm{BE}$ ranging from $\mathrm{ND}$ to AdenoCa:

- ND on first EGD ( $n=644)$ : not a single patient with ND progressed to AdenoCa;

- LGD on first EGD ( $n=968)$;

- 946 LGD (97.7\%) remained with LGD (or bounced: ND/HGD), but did not progress to AdenoCa;

- 13 LGD (1.3\%) progressed through HGD to AdCa (incident AdenoCa from LGD through HGD);

- 10 LGD (1.0\%) progressed directly to AdCa (incident AdenoCa from LGD without HGD);
- HGD on first EGD: $(n=51)$ (prevalent HGD);

- 43 HGD (84.3\%) remained with HGD (or bounced: ND/HGD), but did not progress to AdenoCa; and

- 3 HGD (5.9\%) progressed to AdenoCa (incident AdCa after The Hunt).

\section{Discussion}

Management strategies designed to detect Barrett's cancers must consider several factors:

1. AdenoCa rarely develops in patients with Barrett's and ND.

2. AdenoCa does develop in patients with a diagnosis of only LGD.

3. HGD found in flat Barrett's should be followed by the hunt.

4. With the one-year Hunt, it is guaranteed that your life will be meaningful.

a. $10 \%$ of patients with flat HGD will be harboring a Barrett's cancer that will be detected and cured by your wise decision to employ the hunt.

b. In the 15 years following the hunt, an additional $6 \%$ of patients with HGD found at first EGD will have a Barrett's cancer that will be detected and cured by your wise decision to perform "posthunt" surveillance.

c. Thus, if we find flat HGD during the first EGD, we can expect in the next 15 years that $16 \%$ will have a Barrett's cancer that will be cured as a result of our wisdom and intellectual prowess.

5. With the knowledge that ND is benign and that LGD can precede both HGD and AdCa, you can impress your patients with the following facts:

a. Surveillance during the next 15 years is unlikely to detect anything meaningful if you have ND, but we'll do it anyway because I'm tired of talking about it.

b. Surveillance during the next 15 years will detect curable AdCa in $2 \%$ of all patients with any Barrett's: dysplastic or nondysplastic.

c. Surveillance during the next 15 years will detect curable AdCa in $2.5 \%$ of all patients with dysplastic Barrett's of LGD. 
d. Surveillance during the next 15 years will detect curable AdCa in 3.0\% of all patients with dysplastic Barrett's LGD and HGD. Surveillance during the next 15 years will detect curable AdCa in $19.0 \%$ of all patients who have HGD at any time.

6. Thirty of the 31 patients with AdCa detected on our program were curable, including those whose last EGD was three years earlier.

\section{Conclusions}

If the endoscopist has clearly visualized a flat Barrett's mucosa and has obtained appropriate biopsy specimens:

1. The hunt, followed by surveillance, is a safe and effective protocol for patients with flat HGD.

2. Patients with LGD (without HGD/AdCa) can safely be followed with EGD at two to three year intervals.

3. Patients with HGD should undergo the hunt, followed by surveillance EGD.

4. Yearly EGD (for LGD) and quarterly EGD (for HGD) is not necessary after the first intensive year if the endoscopist has clearly visualized a flat (no bumps) mucosa and has obtained appropriate biopsy specimens.

5. Surgical resection for Barrett's HGD is a thing of the past.

\section{Which biopsy protocol is most likely to detect dysplasia in Barrett's mucosa?}

Sanjay Nandurkar

sanjay.nandurkar@med.monash.edu.au

$\mathrm{BE}$ is a chronic condition characterized by a change in lining of esophageal mucosa from squamous epithelium to columnar metaplasia. The latter epithelium can develop dysplasia, which can lead to the development of adenocarcinoma. Thus, Barrett's is an important preneoplastic condition that confers a 30-50-fold increased risk of adenocarcinoma. The incidence of adenocarcinoma of the esophagus has increased significantly in the last two decades in the Western world.

Periodic endoscopic and histological surveillance has been recommended as a means to detect dysplasia or adenocarcinoma at an early stage, as instituting therapy at this point confers survival ad- vantage. Barrett's segments can vary widely: some can be only a few centimeters long (short segment Barrett's) and others can extend more than $10 \mathrm{~cm}$. Accurate sampling of mucosa to get a good representative pathological perspective of the entire Barrett's segment can pose a challenge. Most gastroenterology societies recommend taking a biopsy from four quadrants at $2 \mathrm{~cm}$ intervals. While this is logistically easy for short segment Barrett's, this can be quite time consuming for long segments.

A recent study from the Glasgow Royal Infirmary analyzed the biopsy practice between two groups of endoscopists; the surgeons adopted the systematic 4-quadrant approach in 1995, whereas the physicians continued to take fewer nonsystematic biopsies. The mean number of biopsies taken per patient was greater in the surgical group compared to the physicians (16 vs. 4 , respectively). And this was evidenced by greater detection of LGD and HGD by the surgeons compared with the physicians (LGD: $18.9 \%$ vs. $1.6 \%$; HGD: $2.8 \%$ vs. $0 \%$ ). Advanced cancer was seen in three cases in the physicians group but none in the surgical group. ${ }^{14}$ This study underscores the importance of a systematic approach to surveillance. Apart from not taking adequate biopsy samples, the other significant issue is the lack of follow up of many patients with BE. Data from a VA center in the United States analyzed a cohort of 472 patients with Barrett's between 1995 and 2005. They found that two-thirds of patients $(n=305)$ had only one endoscopy performed, and approximately $25 \%$ of patients with LGD missed their surveillance interval by more than six months. ${ }^{15}$

In spite of an adequate number of biopsies per protocol, endoscopic sampling only examines a very limited surface area. The standard biopsy forceps can obtain a $1.9 \mathrm{~mm}$ biopsy (with a depth of 1 $\mathrm{mm}$ ). Biopsy forceps with a larger cup (jumbo forceps) can increase the surface area sampled (width $=$ $3.3 \mathrm{~mm}$, depth $=2 \mathrm{~mm}$ ). Intuitively, it would appear more logical to use jumbo forceps to obtain greater tissue mass for analysis. One small study has shown improved dysplasia detection using jumbo forceps, but larger studies are needed. ${ }^{16}$ There is good evidence that has shown that mucosal irregularity, nodules, and ulcers with the Barrett's segment are highly associated with the presence of dysplasia and/or cancer. Thus, close scrutiny of the Barrett's 
segment to identify surface irregularity and getting targeted biopsies from those areas is extremely important before embarking on 4-quadrant biopsies, lest those landmarks get obliterated by blood during sampling.

The presence of dysplasia, especially HGD, portends the presence of concomitant adenocarcinoma or the possibility of cancer developing in the immediate future. Thus, patients with HGD need closer observation at frequent intervals. Although some groups have campaigned for early resection in HGD to avoid missing a prevalent cancer, the Seattle group has shown quite convincingly that adequate biopsy sampling at close time intervals does not lead to missed cancers. ${ }^{17}$ They advocated taking 4 -quadrant biopsies at $1-\mathrm{cm}$ intervals using jumbo forceps as well as taking multiple biopsies from any macroscopic abnormality. They showed that in patients with HGD who have a high probability of harboring cancer, their $1-\mathrm{cm}$-interval protocol detected all cancers, whereas a $2-\mathrm{cm}$ protocol would have missed $50 \%$ of the cancers. The Cleveland group performed a retrospective analysis and found that in their hands the $1-\mathrm{cm}$ protocol and the standard $2-\mathrm{cm}$ protocol both performed badly and missed $30-40 \%$ of intramucosal cancers (although no submucosal cancers escaped detection). However, Cleveland study has been criticized by some as being retrospective for proactively identifying and targeting macroscopic irregularities that the Seattle group highlighted.

The use of acetic acid improves surface visualization and is quick and easy to apply. Preliminary data suggest that acetic acid target biopsies improve detection of dysplasia compared to the standard 2$\mathrm{cm}$ protocol. Numerous investigators have evaluated the use of chromoendoscopy (usually methylene blue) to identify suspicious areas for targeted biopsies. However, it appears to be highly operator dependent, and the published reports are highly contradictory; hence, its use would difficult to advocate.

Newer imaging techniques such as narrow band imaging (NBI) and Fujinon intelligent color enhancement (FICE) can provide improved visualization of surface mucosa. There is a significant amount of published literature that has shown improvement in neoplasia detection using NBI technology. A recent meta-analysis of eight studies that included 446 patients and 2,194 lesions showed that the sen- sitivity and specificity for detection of HGD using NBI was 0.96 and 0.94 , respectively. ${ }^{18}$ Confocal laser endomicroscopy (CLE) is a revolutionary technique that images subcellular tissue in real time (virtual biopsy). However, it samples only a highly limited surface area, is time consuming, and early reports to date have not shown it to be better than standard biopsy protocol.

In summary, scheduled surveillance protocol with $2-\mathrm{cm}$ protocol for Barrett's patients at average risk and $1-\mathrm{cm}$ protocol (Seattle) for patients at high risk shows the best outcome. Adequate visualization of Barrett's surface mucosa is critical to identify mucosal irregularities which are associated with HGD and or cancer. Targeted biopsies using acetic acid and NBI and use of jumbo forceps to obtain larger samples appears to be beneficial. Use of chromoendoscopy cannot be routinely advocated, except in certain institutions.

\section{Can gastric atrophy be an explanation of the inverse association of adenocarcinoma with $H$. pylori infection?}

\author{
Hala El-Zimaity \\ hala.el-zimaity@uhn.on.ca
}

Until the mid 1970s, adenocarcinoma was a rare cancer type in the esophagus. Since then, the continuing decline in $H$. pylori infection in Western populations has been associated with a marked increase in GERD, BE, and EAC. ${ }^{19}$ The question to answer here is, "can gastric atrophy be an explanation of the inverse association of adenocarcinoma with H. pylori infection?"

The explanation for the inverse association would come from clinical trials. Epidemiologic data suggest that a subset of $H$. pylori-infected patients may experience a lower risk of Barrett's carcinoma. Many studies use serology biomarkers to screen patients for gastric atrophy. Serum pepsinogen I is an indirect measure of corpus function. As PGI is secreted only by oxyntic glands, with advancing corpus atrophy, PG1 goes down. A low sPGI is a serologic marker of corpus atrophy. Most of Japanese Barrett's patients are $H$. pylori naïve with high serum pepsinogen levels. ${ }^{20} \mathrm{H}$. pylori infection was present in four of 36 patients $(11 \%)$ with Barrett's and in 80 of 108 controls $(74 \%, P<0.0001) .^{20}$ In 
H. pylori-negative subjects, both serum pepsinogen I and pepsinogen II concentrations are significantly higher in Barrett's patients than in controls (mean pepsinogen I: Barrett's $51.0-14.0 \mathrm{ng} / \mathrm{mL}$ vs. control $38.9-13.5 \mathrm{ng} / \mathrm{mL}, P=0.0012$; mean pepsinogen II: Barrett's $10.8-4.0 \mathrm{ng} / \mathrm{mL}$ vs. control $7.9-2.0 \mathrm{ng} / \mathrm{mL}, P=0.0097)$. Thus, in Japan, an inverse association exists between low pepsinogen I levels (a marker of advanced gastric atrophy) and Barrett's. $^{20}$

Since 1969, Japanese physicians have used endoscopy to visualize changes in the gastric mucosa of gastritis patients. ${ }^{21}$ Atrophic gastric mucosa is pale yellowish in color, with transparent blood vessels, and nonatrophic mucosa is homogeneously reddish and smooth. The atrophic border is the boundary between the antral and fundic glandular territories. At endoscopy, the atrophic border is recognized by discriminating between the differences in the color and height of the gastric mucosa. Japanese physicians incorporated the endoscopic atrophic border in an endoscopic grading system for atrophy. ${ }^{21}$ Corpus atrophy begins at atrophic border (antrumcorpus border) in particular the incisura and extends proximally more rapidly up the lesser curve than the greater curvature. Atrophy in corpus biopsies high on the greater curvature are among the last to show atrophy. Following the Japanese endoscopic grading system, atrophy limited to the lesser curve is "closed." Atrophy extending high on the greater curvature is "open." Kim et al. showed the endoscopic grading of atrophic gastritis is inversely associated with gastroesophageal reflux and gastropharyngeal reflux. ${ }^{22}$ GERD and gastropharyngeal reflux disease was significantly lower in the open type (advanced gastric atrophy) than in the closed type (lessadvanced gastric atrophy $(P<0.001, P=0.012$, respectively). ${ }^{22}$

The endoscopic grading system shows a good correlation with histological evaluation. Gastric background mucosa in patients with distal esophageal cancer has a higher prevalence of nonatrophic gastric mucosa when compared to gastric background mucosa in patients with distal gastric cancer. The latter are always associated with advanced gastric atrophy. ${ }^{23}$ Altogether, studies suggest that gastric atrophy associated with $H$. pylori infection may be responsible for the inverse association between $H$. pylori infection and esophageal disease.

\section{What are the arguments against the decrease of the risk of BE by $H$. pylori infection?}

\section{Angel Lanas}

alanas@unizar.es

H. pylori infection has been associated with a decreased risk of GERD and related diseases. No clinical trials are available to answer this question. In general, epidemiological studies have found an inverse relationship between $H$. pylori infection and/or CagA-positive strains and BE and adenocarcinoma of the esophagus, but these findings have not been consistent in all studies, and two meta-analyses have come out with different conclusions. One, reported in 2007, concluded that in patients with BE there were inverse relationships with $H$. pylori infection overall and with Cag-A-positive strain (OR, 0.64; 95\% CI, 0.43-0.94; OR, 0.39; 95\% CI, 0.21-0.76, respectively). ${ }^{24}$ However, a most recent one with a higher number of studies ${ }^{25}$ concluded that there was no association between $H$. pylori infection and BE (Fig. 2). The overall prevalence of $H$. pylori infection between $\mathrm{BE}$ and controls was $42.9 \%$ versus $43.9 \%$ (OR $=0.74,95 \%$ CI 0.40-1.37), but with significant heterogeneity. Further analyses were conducted based on the type of controls used as reference group. In nine studies, the prevalence of $H$. pylori infection was lower in BE than in endoscopically normal healthy controls $(23.1 \%$ vs. $42.7 \%$, OR $=0.50,95 \%$ CI $0.27-0.93)$ with significant heterogeneity observed between studies. In contrast, $H$. pylori infection was significantly increased in BE patients in the three studies using healthy blood donors as "normal controls" (71.2\% vs. $48.1 \%, \mathrm{OR}=2.21,95 \%$ CI $1.07-4.55$ ).

The question then may rely on who is the appropriate control for this type of studies. In principle, patients undergoing endoscopy for different indications should be excluded as controls since they may suffer of diseases linked to $H$. pylori infection. On the other hand, blood donors may represent a selected population. Controls should be as close as possible to the general healthy population and should be matched by age and sex, since $H$. pylori infection prevalence is age dependent, and $\mathrm{BE}$ is more frequent in males. Other factors that should be considered when interpreting these studies are geographical/race differences, definitions of 
Outcome: Prevalence of H. pylori infection in BE and healthy controls

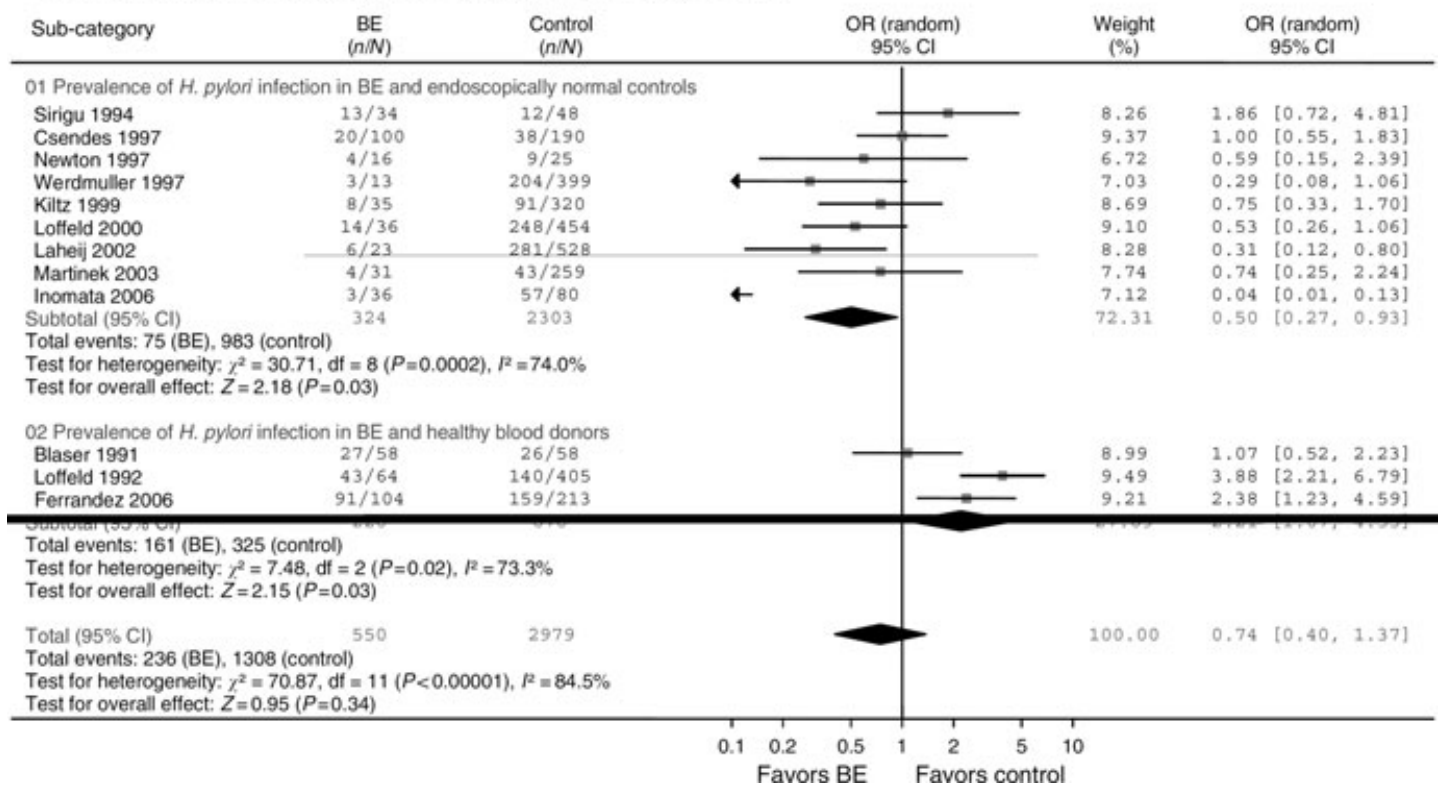

Figure 2. Meta-analysis of the prevalence of Helicobacter pylori in patients with BE versus controls. ${ }^{25}$ With permission from Macmillan Publishers Ltd.

$\mathrm{BE}$, using a retrospective versus a prospective approach, and the diagnostic tests used for $H$. pylori infection. Furthermore, confounding variables can explain negative results. No clear mechanistic explanation is available. The most frequent one has been linked to a reduced acid output due to the presence of corpus gastritis and different degrees of atrophy in H. pylori-infected individuals, but a negative association has been found in patients with negative atrophic gastritis. Also, H. pylori infection may increase gastric secretion in patients with antral gastritis. ${ }^{26,27}$ Therefore, due to the low quality of most studies and the lack of an appropriate mechanistic explanation, we cannot conclude that there is a relationship between H. pylori and BE. Well-performed, larger studies with appropriate controls matched by age and sex should be conducted.

\section{Should decrease of PPI efficacy following eradication of $\boldsymbol{H}$. pylori be considered in the treatment of BE?}

Angel Lanas

alanas@unizar.es

It has been shown that PPIs induce a higher degree of acid inhibition in $H$. pylori-infected in- dividuals, which provides higher healing rates in erosive esophagitis patients treated with PPIs. ${ }^{28} \mathrm{~Pa}$ tients with $\mathrm{BE}$ are recommended to be treated with high-dose PPI to reduce gastric acid and reduce esophageal acid exposure as much as possible. Patients with BE can undergo $H$. pylori eradication under different circumstances. This may reduce the capacity of PPIs to inhibit acid gastric acid secretion. However, no studies have addressed specifically this question in BE patients. There are no studies to define whether $H . p y$ lori eradication worsens or improves $\mathrm{BE}$ after H. pylori eradication. In one study, which included 24 cases of short-segment BE within a sample of 82 patients with peptic ulcer disease, it was shown that after $H$. pylori eradication, there were six new cases of short-segment BE after a mean follow-up of 24 months. ${ }^{29}$ Another study showed that $H$. $p y$ lori infection did not influence esophageal acid reflux and symptoms in patients with BE, either at baseline or during low, as well as profound, acid suppressive therapy. The authors concluded that the dose of acid suppression does not have to be titrated upon $H$. pylori status in GERD patients (Fig. 3). ${ }^{30}$ A systematic review of 27 studies concluded that $H$. pylori eradication in peptic ulcer patients does not induce esophagitis or worsen 


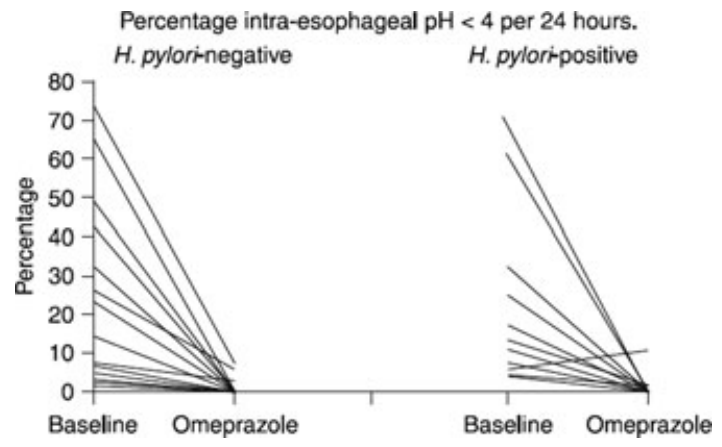

Figure 3. Individual values of percentage intraesophageal $\mathrm{pH}$ $<4$ per 24 hours in Helicobacter pylori-negative and $H$. $p y$ lori-positive Barrett's esophagus patients at baseline and during therapy with omeprazole $40 \mathrm{mg}$ b.d. at three months. ${ }^{30} \mathrm{With}$ permission from John Wiley \& Sons.

GERD symptoms. ${ }^{31}$ Also, it has been shown that patients with established GERD did not require increased doses of PPI after H. pylori eradication. ${ }^{32}$

Therefore, although no studies have specifically addressed this question in BE patients, indirect evidence suggests that a potential decrease of PPI efficacy should not preclude the indication for $H$. pylori eradication, and that this should not be of concern for patients with BE.

\section{Is surgical treatment likely to modify the progression of natural history of BE?}

Paolo Parise

p.parise@ao-pisa.toscana.it

The American College of Gastroenterology 2008 guidelines defines $\mathrm{BE}$ as a "change in distal esophageal epithelium of any length that can be recognized as columnar type mucosa at endoscopy and is confirmed to have intestinal metaplasia by biopsy of the tubular esophagus." ${ }^{33}$ Patients with long-segment BE have a 30-125-fold increased risk of developing esophageal cancer, ${ }^{34,35}$ but the real natural history of the sequence metaplasiadysplasia-carcinoma is still unclear, because most of these patients undergo surgical or medical therapy. However, in this multistep sequence, dysplasia represents the best marker of the development of adenocarcinoma of the gastro-esophageal junction. In this cascade, some steps are thought to be very rarely reversible (BE normal epithelium) or never (adenocarcinoma-dysplasia), but others can and do regress. This represents the rationale for the surveillance and medical or surgical treatment of BE.

In a prospective study in 1999, Weston and colleagues followed 108 patients affected by $\mathrm{BE}$ and who were treated with PPI for a mean period of 40 months. At baseline endoscopy, they found 80 patients with ND, 20 with LGD, and eight with HGD. Among the nondysplastic group, $2.5 \%$ progressed to cancer and $28.7 \%$ progressed to LGD with a subsequent regression rate of $78.2 \%$. In the LGD group, $65 \%$ of patients regressed to ND, but $20 \%$ progressed to HGD, subsequently developing cancer or multifocal HGD in 50\% cases and nondysplastic $\mathrm{BE}$ in the remaining $50 \%$ cases. Patients presenting with HGD developed cancer or multifocal HGD in $62.5 \%$ cases and regressed to LGD or ND in the other cases. ${ }^{36}$

In a retrospective study from the Netherlands, Hage and colleagues analyzed 105 patients affected by at least $3 \mathrm{~cm}$ BE without HGD or cancer at first endoscopy for a mean follow-up period of 12.7 years. LGD was present in 11 patients at entry endoscopy. At the end of follow-up, 6\% patients had developed adenocarcinoma, which equals one cancer case per 221 patient-year or $0.45 \%$ per year. Tumors were interestingly diagnosed in a very wide range of time, from 4.6 to 15.9 years after index endoscopy. HGD was diagnosed in $5 \%$ of patients, which equals one cancer case per 266 patient-year or $0.38 \%$ per year. Similarly, diagnosis was made in wide period, ranging from 0.6 to 18.9 years. Regression from LGD to nondysplastic Barrett's epithelium was observed in $50 \%$ cases, but a progression to HGD was observed in $25 \%$ cases. A trend towards cancer development was observed for increasing lengths of BE. ${ }^{37}$ But, with logistic regression analysis, other risk factors have been identified for development of multifocal HGD or adenocarcinoma: dysplasia at first endoscopy $(P<0.001),>3 \mathrm{~cm}$ hiatal hernia $(P<0.02)$, presence of dysplasia at any time during follow-up $(P<0.03)$, and Barrett's epithelium $>2 \mathrm{~cm}(P<0.009) .{ }^{36}$

The rationale of laparoscopic antireflux surgery in patients affected by BE is to prevent the progression to dysplasia-carcinoma and even to induce the regression to a nondysplastic Barrett's epithelium or a non metaplastic epithelium. It is commonly accepted that, once established, BE doesn't regress. This conclusion is supported by some endoscopic data that show no change in the length of the 
columnar-lined epithelium under therapy, ${ }^{38}$ but in a study on 77 patients treated with antireflux surgery versus 14 patients treated with PPI, Gurski et al. observed a complete regression of the metaplastic epithelium in $36.4 \%$ of surgical patients versus $7.1 \%$ of medical patients $(P<0.03)$. Authors also evidenced that regression happened only in patients with short segment BE. ${ }^{39}$ Even better results were reported in a recent study on 125 patients with short segment BE treated with laparoscopic Nissen fundoplication, duodenal switch, or duodenal diversion. Regression to nonmetaplastic mucosa was observed in $61 \%$ patients of the Nissen group, and $64 \%$ and $65 \%$, respectively, in the other two groups. ${ }^{40}$ Antireflux surgery also seems to be effective in inducing regression from LGD and preventing progression to HGD or cancer. Hofstetter and colleagues evaluated the long-term outcome (five years) of antireflux surgery in 97 patients affected by BE. LGD regressed to nondysplastic Barrett's in $44 \%$ cases and intestinal metaplasia was lost, regressing to cardiac mucosa, in $14 \%$. No patient developed HGD or cancer in 410 patient-years of follow-up, but LGD developed in $6 \%$ patients. ${ }^{41}$ In 2003, Parrilla published the results of a randomized study comparing medical and surgical treatment of BE patients. In the medical group, 2 of 3 patients regressed from LGD to ND, and in the surgical group, 5 of 5 regressed. In the medical arm, dysplasia de novo appeared in 8 of 40 patients (20\%); two of these developed HGD. In the surgical arm, 3 of 53 patients $(6 \%)$ developed dysplasia de novo, and two of these subsequently progressed to HGD, but these two showed a $\mathrm{pH}$-metric recurrence of gastroesophageal reflux. No statistically significant differences were found between these two groups. If the patients with $\mathrm{pH}$-metric failure are excluded, dysplasia de novo appeared only in $2 \%$ of patients and the surgical group showed a statistically significant difference respect the medical group $(P<$ $0.05)$. The rate of malignancy was $0.8 \%$ per year in the medical group and $0.5 \%$ in the surgical group. ${ }^{42}$ Similar good results were obtained in a little study from our institution, where medical (19 patients) versus surgical (16 patients) treatment were compared in LGD patients at 18 months follow-up. We observed a regression rate of $63.2 \%$ in the medical arm versus a $93.8 \%$ in the surgical arm $(P=0.047)$. The association between treatment and remission of LGD was examined using multiple logistic regression; laparoscopic Nissen fundoplication was the only variable associated with the probability of remission of LGD. ${ }^{43}$

These apparently better results of surgery respect medical therapy could be explained by the fact that the esophageal mucosa exposure to duodeno-gastric refluxate is higher in patients with $\mathrm{BE}$ and with dysplasia $^{44}$ and PPI are not able to block bile reflux; moreover, we know that even under PPI therapy about $60 \%$ patients have pathological esophageal exposure to bile, in a weakly acid environment that promotes the deconjugation of bile-salts. ${ }^{45}$ Unfortunately, these results are not completely confirmed by large meta-analysis of the literature. ${ }^{46,47}$ In fact, surgically treated patients demonstrated a higher incidence of regression from LGD to Barrett's epithelium (15.4\%) when compared with medically treated patients (1.9\%). Even when only controlled studies were analyzed, the probability of developing regression was greater in the surgically treated group (6.5\% vs. $0.5 \%, P=0.024)$.

The largest difference between surgical and medical therapy was demonstrated in the probability of regression from nondysplastic BE to normal squamous epithelium (17\% vs. $0.4 \%)$. But, in regard of incidence of EA, when data from all included studies are pooled, the median incidence of cancer was 2.8 cases per 1,000 patient-years among the surgical group and 6.3 in the medical group $(P=0.034)$. When data from only controlled studies were pooled, the median incidence of adenocarcinoma in the surgical group did not significantly differ from that of the medical group: 4.8 cases per 1,000 patient-years versus 6.5 , respectively $(P=0.32)$.

These conflicting results between the risk of cancer development and induction of regression could be explained with the fact that cellular and genetic alterations leading to adenocarcinoma may have already occurred before an antireflux procedure was performed; thus some cancers, particularly those that present during the first few postoperative years, probably do not represent progression of the disease.

Even though antireflux surgery seems to better promote regression of $\mathrm{BE}$ or dysplasia than medical therapy, it has not demonstrably reduced the incidence rate of adenocarcinoma, and therefore cannot be currently recommended as an antineoplastic procedure. Nevertheless, antireflux procedures should be proposed as a therapeutic alternative to all patients affected by BE with or without 
associated dysplasia, thus balancing the risks and benefits for each patient.

\section{Is trimodal esophageal imaging (HRE-FI-NBI) the best tool for enhancement of detection of early neoplasia in Barrett's patients?}

\section{René Lambert}

lambert@iarc.fr

Columnar metaplasia in the esophagus (Barrett's) occurs in relation to esophageal reflux and obesity in the young age. Three distinct types of gastric epithelium may develop in the distal esophagus: a cardiac type with mucous epithelial cells, a fundic type with chief and oxyntic cells, and an intestinal type with goblet cells and enterocytes with a brush border. The prevalence of Barrett's is higher in Caucasian males of Western countries; however, it has been overestimated, and based on a Swedish population of endoscopies performed in adults, it is now estimated at $1.6 \%$. Multiple studies confirm an increased risk of adenocarcinoma in columnar metaplasia of the esophagus, but it has also overestimated, and recent evaluations account for one case per 200 patient-years. The risk has been correlated with the presence of intestinal cells in the metaplastic segment. However, recent studies conducted in the United States have shown that intestinal cells are found in only $48 \%$ of cases of Barrett's, and a study of small esophageal resected carcinomas has shown that the tumor develops more often $(70 \%)$ in the vicinity of a cardiac type of epithelium. The risk of cancer is the same in the presence or absence of intestinal cells in the metaplastic segment; therefore, endoscopic exploration should aim to detect dysplasia rather than the intestinal type of epithelium.

In Western countries, attention given to the detection of early cancer in BE is justified by an increasing incidence of EA in recent decades; ${ }^{48}$ as an example, the annual variation reaches $+8.6 \%$ in men in the SEER registries of the United States during 1973-1995, but the worldwide incidence of adenocarcinoma is still very low as compared to that of squamous cell cancer in the esophagus. Overall, detection of early esophageal neoplasia in asymptomatic persons aims for curative treatment by endoscopic resection or by radiofrequency. As a rule, early neoplasia in the metaplastic mucosa of a $\mathrm{BE}$ is often missed during endoscopy, even with the help of chromoscopy, when a dye solution is projected at the surface of the mucosa (indigocarmine, methylene blue, or acetic acid). The poor efficacy of targeted biopsies explains the recommendation to adopt the time-consuming Reid protocol, with blind or random biopsies performed in quadrants at each length of $1 \mathrm{~cm}$ in the segment with columnar metaplasia.

In recent years, the dramatic progression in endoscopic imaging, and the cumulative impact of high resolution imaging (HRE), magnification, autofluorescence spectroscopy (AFI), and image processing modifies the diagnostic strategy of neoplasia in BE.

1. High-resolution imaging depends on the increased number of pixels in the charged coupled device (CCD) receiving the efferent photons and on the high (1,080 lines) definition of the image transmitted to the TV receptor.

2. Magnification with an optical zoom at a power of $\times 60$ or $\times 80$ describes with precision the microarchitecture of surface epithelium (pit pattern) and subepithelial capillaries (vascular pattern). An electronic zoom may also contribute to magnification.

3. Autofluorescence imaging requires a specific material: the AFI system proposed by Olympus Medical Systems consists of a light source (XCLV-260HP), a processor (XCV-260HP), a video monitor, and a dedicated videoendoscope (XCF-Q240FAI), which incorporates two CCDs, one for autofluorescence, the other for white light. In autofluorescence, the excitation light $(390-470 \mathrm{~nm})$ is provided by a rotation filter on the light source. The resulting image is artificially colored to green. A normal mucosa emits a bright autofluorescence and is colored in green. Fluorophores in the tumor absorb autofluorescence; the lesion appears in the complementary color of green-magenta. However, false-positive reactions in magenta are common.

4. Image processing with the NBI technique of Olympus is based on the restriction of the afferent light in two channels (blue and green); in the efferent image the micro-architecture of the epithelial crest is enhanced, and the subepithelial capillaries have greater contrast. Other methods in image processing have been developed by Fujinon (FICE) and Pentax (i-scan). 
The increased efficacy of HRE endoscopy in white light combined to NBI has been stressed; both are proposed as red flag techniques for the detection of neoplasia in Barrett's. ${ }^{49,50}$ Recently, a further progress in sensitivity and specificity is linked to the trimodal technology, combining HRE, AFI, and NBI, with or without magnification. ${ }^{51,52}$ This protocol is proposed in a joint venture by endoscopy units based in the Netherlands (Amsterdam) and England (Manchester). The trimodal protocol significantly increases the sensitivity of HRE and more positive results are found. In addition, NBI reduces the false-positive rate of HRE + AFI from $71 \%$ to $48 \%$; however, it is concluded that this protocol cannot substitute for the blind and random biopsies protocol in BE. ${ }^{52}$

In conclusion, the trimodal protocol requires specialized materials, is restricted to some reference centers, and, in Western countries, random biopsies are still recommended in asymptomatic patients with $\mathrm{BE}$ for the detection of neoplasia. However, in those countries, not enough room is attributed to magnification. Currently, in Japan, the detection of suspicious areas at the surface of the mucosa is completed by characterization through categories of the pit pattern and vascular pattern, and the best method of detection of flat areas of neoplasia relies on a bimodal HRE-NBI protocol coupled with magnification. Both protocols are currently available in this country with video-endoscopes and deserve to be spread around the world. Exploration of BE should rely on a protocol ensuring characterization after detection, while targeted biopsies could replace random biopsies.

\section{The essential importance of natural history for the wise management of HGD}

\section{Helen M. Shields \\ hshields@caregroup.harvard.edu}

HGD is not a fixed, well-defined entity. This is a major problem to address in the management of HGD in BE. Poor interobserver reproducibility is present in the diagnosis of HGD even when excellent pathologists, experienced in reading gastrointestinal biopsies, focus on diagnosing HGD on Barrett's slides from Barrett's patients. ${ }^{53}$ Dr. Maru from the M.D. Anderson Cancer Center notes that $41 \%$ of outside cases called "HGD" are classified as adenocarcinoma by their group of pathologists. ${ }^{53}$ It is important to recognize this fact in making a decision about what is the best treatment for HGD.

Two very divergent studies have assessed the risk of HGD in BE patients turning into adenocarcinoma ${ }^{54,55}$ In the Hines VA study, Schnell et al. noted $16 \%$ of patients evolving to adenocarcinoma over a follow-up interval of seven years and three months of surveillance endoscopies. ${ }^{55}$ On the other hand, Reid's group at the University of Washington noted that $59 \%$ of their HGD patients had developed adenocarcinoma at the five-year time interval. ${ }^{54}$ This marked divergence in the evolution of HGD to adenocarcinoma leads to significant doubt about the reproducibility of the diagnosis of HGD as supported by Maru's observations above. ${ }^{53}$ What should be done for patients with HGD that has been verified by at least two experienced pathologists? The most effective and least harmful therapy should be recommended. Esophagectomy has traditionally been associated with significant morbidity and mortality. It is currently reserved for patients who are not considered candidates for less invasive, but potentially curative therapies, such as photodynamic therapy, endoscopic mucosal resection, and/or radiofrequency ablation. ${ }^{56,57}$ Photodynamic therapy's relatively high rate of stricture formation and photosensitivity have limited its role now that other modalities are available.

Ell et al. pointed out the feasibility of endoscopic mucosal resection for HGD and early cancer in 64 BE patients with early esophageal cancer or HGD. ${ }^{56}$ The best evidence for cure available at present is the use of endoscopic mucosal resection. ${ }^{56}$ Another excellent endotherapy is radiofrequency ablation. The results reported in the 2009 New England Journal of Medicine paper from Shaheen et al. showed that the radiofrequency ablation method has its greatest benefit in patients with HGD. Patients were randomly assigned to receive the actual treatment or a sham procedure. Eradication of HGD was found in $81 \%$ of the group receiving the therapy ${ }^{57}$ Compared to historical reports of the natural history of $\mathrm{BE}$, ablation may be associated with a reduction in cancer incidence. But heterogeneous studies limit the comparison. A frequently asked question is whether radiofrequency ablation should be added to endoscopic mucosal resection to improve follow-up results. The answer is not known at present. Also, the role of laparoscopic or traditional surgery to cure 
an EA has not been compared to endoscopic mucosal resection or radiofrequency ablation to date in a randomized controlled trial. These prospective studies are needed.

In summary, the wise management of HGD is based on understanding that there is variability in the diagnosis of HGD by pathologists. At present, endoscopic mucosal resection therapy with or without radiofrequency ablation appears to be reasonable therapy for HGD or early esophageal carcinoma. Research data also favor the use of radiofrequency ablation compared to photodynamic therapy because of fewer complications and better efficacy. However, neither endoscopic mucosal resection nor radiofrequency ablation has been studied in randomized controlled trials and compared to surgical therapy. Long-term follow-up data are needed for all forms of therapy.

\section{Conflicts of Interest}

The authors declare no conflicts of interest.

\section{References}

1. Kahrilas, P.J. \& J.E. Pandolfino. 2002. Gastroesophageal reflux disease and its complications, including Barrett's metaplasia. In Sleisenger and Fordtran's Gastrointestinal and Liver Disease: Pathophysiology/Diagnosis/Management. Chapter 33. M. Feldman, L.S. Friedman \& M.H. Sleisenter, Eds.: 616. Saunders. Philadelphia.

2. Spechler, S.J., R.C. Fitzgerald, G.A. Prasad \& K.K. Wang. 2010. History, molecular mechanisms, and endoscopic treatment of Barrett's esophagus. Gastroenterol 138: 854-859.

3. Society of Thoracic Surgeons, website, http://www.sts. org/.2010.

4. Blot, W.J. \& J.F. Fraumeni, Jr. 1987. Trends in esophageal cancer mortality among US blacks and whites. Am. J. Public Health 77: 296-298.

5. Polednak, A.P. 2003. Trends in survival for both histologic types of esophageal cancer in US surveillance, epidemiology and end results areas. Int. J. Cancer 105: 98-100.

6. Camilleri, M., D. Dubois, B. Coulie, et al. 2005. Prevalence and socioeconomic impact of upper gastrointestinal disorders in the United States: results of the US Upper Gastrointestinal Study. Clin. Gastroenterol. Hepatol. 3: 543-552.

7. Hayeck, T.J., C.Y. Kong, S.J. Spechler, et al. The prevalence of Barrett's esophagus in the US: estimates from a simulation model confirmed by SEER data. Dis. Esophagus, 23: 451-457.

8. Gerson, L.B., R. Edson, P.W. Lavori \& G. Triadafilopoulos. 2001. Use of a simple symptom questionnaire to predict Barrett's esophagus in patients with symptoms of gastroesophageal reflux. Am. J. Gastroenterol. 96: 2005-2012.

9. Ronkainen, J., P. Aro, T. Storskrubb, et al. 2005. Prevalence of Barrett's esophagus in the general population: an endoscopic study. Gastroenterology 129: 1825-1831.

10. Oberg, S., J. Johansson, J. Wenner \& B. Walther. 2002. Meta- plastic columnar mucosa in the cervical esophagus after esophagectomy. Ann. Surg. 235: 338-345.

11. Su, Y., X. Chen, M. Klein, et al. 2004. Phenotype of columnarlined esophagus in rats with esophagogastroduodenal anastomosis: similarity to human Barrett's esophagus. Lab. Invest. 84: 753-765.

12. Souza, R.F., K. Krishnan \& S.T. Spechler. 2008. Acid, bile, and CDX: the ABCs of making Barrett's metaplasia. Am. J. Physiol. Gastrointest. Liver Physiol. 295: G211-G218.

13. Alvaro-Villegas, J.C., S. Sobrino-Cossio, A. HernandezGuerrero, et al. Dilated intercellular spaces in subtypes of gastroesophagic reflux disease. Rev. Esp. Enferm. Dig. 102: 302-307.

14. Abela, J.-E., J.J. Going, J.F. Mackenzie, et al. 2008. Systematic four-quadrant biopsy detects Barrett's dysplasia in more patients than nonsystematic biopsy. Am. J. Gastroenterol. 103: 850-855.

15. Ajumobi, A., K. Bahjri, C. Jackson \& R. Griffin. 2010. Surveillance in Barrett's esophagus: an audit of practice. Dig. Dis. Sci. 55: 1615-1621.

16. Komanduri, S., G. Swanson, L. Keefer \& S. Jakate. 2009. Use of a new jumbo forceps improves tissue acquisition of Barrett's esophagus surveillance biopsies. Gastrointest. Endosc. 70: 1072-1078.

17. Reid, B.J., P.L. Blount, Z. Feng \& D.S. Levine. 2000. Optimizing endoscopic biopsy detection of early cancer in Barrett's high-grade dysplasia. Am. J. Gastroenterol. 95: 3089-3096.

18. Manath, J., V. Subramanian, J. Hawkey \& K. Ragunath. 2010. Narrow band imaging for characterization of high-grade dysplasia and specialized intestinal metaplasia in Barrett's esophagus: a meta analysis. Endoscopy 42: 351-359.

19. Blaser, M.J. 1999. Hypothesis: the changing relationships of Helicobacter pylori and humans: implications for health and disease. J Infect Dis. 179: 1523-1530.

20. Abe, Y., K. Iijima, T. Koike, et al. 2009. Barrett's esophagus is characterized by the absence of Helicobacter pylori infection and high levels of serum pepsinogen I concentration in Japan. J. Gastroenterol. Hepatol. 24: 129-134.

21. Kimura, K. 1973. Chronological changes of atrophic gastritis. Nippon Shokakibyo Gakkai Zasshi. 70: 307-315.

22. Kim, D.H., G.H. Kim, J.Y. Kim, et al. 2007. Endoscopic grading of atrophic gastritis is inversely associated with gastroesophageal reflux and gastropharyngeal reflux. Korean J. Intern. Med. 22: 231-236.

23. El-Zimaity, H.M., H. Ota, D.Y. Graham, et al. 2002. Patterns of gastric atrophy in intestinal type gastric carcinoma. Cancer 94: 1428-1436.

24. Rokkas T., D. Pistiolas, P. Sechopoulos, et al. 2007. Relationship between Helicobacter pylori infection and esophageal neoplasia: a meta-analysis. Clin Gastroenterol. Hepatol. 5: 1413-1417.

25. Wang, C., Y. Yuan \& R.H. Hunt. 2009. Helicobacter pylori infection and Barrett's esophagus: a systematic review and meta-analysis. Am. J. Gastroenterol. 104: 492-500.

26. Mccoll, K.E. 2007. Helicobacter pylori and oesophageal cancer-not always protective. Gut. 56: 457-459.

27. Axon, A.T. 2004. Personal view: to treat or not to treat? Helicobacter pylori and gastro-oesophageal reflux diseasean alternative hypothesis. Aliment. Pharmacol. Ther. 19:253261. 
28. Holtmann, G., C. Cain \& P. Malfertheiner. 1999. Gastric Helicobacter pylori infection accelerates healing of reflux esophagitis during treatment with the proton pump inhibitor pantoprazole. Gastroenterology 117: 11-16.

29. Yachida, S., D. Saito, T. Kozu, et al. 2001. Endoscopically demonstrable esophageal changes after Helicobacter pylori eradication in patients with gastric disease. J. Gastroenterol. Hepatol. 16: 1346-1352.

30. Peters, F.T., E.J. Kuipers, S. Ganesh, et al. 1999. The influence of Helicobacter pylori on oesophageal acid exposure in GERD during acid suppressive therapy. Aliment. Pharmacol. Ther. 13: 921-926.

31. Raghunath, A.S., A.P. Hungin, D. Wooff \& S. Childs. 2004. Systematic review: the effect of Helicobacter pylori and its eradication on gastro-oesophageal reflux disease in patients with duodenal ulcers or reflux oesophagitis. Aliment. Pharmacol. Ther. 20: 733-744.

32. Kuipers, E.J., G.F. Nelis, E.C. Klinkenberg-Knol, et al. 2004. Cure of Helicobacter pylori infection in patients with reflux oesophagitis treated with long term omeprazole reverses gastritis without exacerbation of reflux disease: results of a randomised controlled trial. Gut. 53: 12-20.

33. Wang, K.K. \& R.E. Sampliner. 2008. Updated guidelines 2008 for the diagnosis, surveillance and therapy of Barrett's esophagus. Am. J. Gastroenterol. 103: 788-797.

34. Spechler, S.J., A.H. Robbins, et al. 1984. Adenocarcinoma and Barrett'esophagus: an overrated risk? Gastroenterology 87: 927-933.

35. Cameron, A.J., B.J. Ott, et al. 1985. The incindence of adenocarcinoma in columnar-lined (Barrett's) esophagus. N. Engl. J. Med. 313: 857-859.

36. Weston, A.P., A.S. Badr, et al. 1999. Prospective multivariate analysis of clinical, endoscopic, and histological factors predictive of the development of Barrett's multifocal highgrade dysplasia or adenocarcinoma. Am. J. Gastroenterol. 94: 3413-3419.

37. Hage, M., D. Siersema, et al. 2004. Oesophageal cancer incidence and mortality in patients with long-segment Barrett's oesophagus after a mean follow-up of 12,7 years. Scand. J. Gastroenterol. 12: 1175-1179.

38. Peters, F.T., S. Ganesh, et al. 1999. Endoscopic regression of Barrett's esophagus during omeprazole treatment: a randomized double-blind study. Gut. 45: 489-494.

39. Gurski, R., J.H. Peters, et al. 2003. Barrett's esophagus can and does regress after antireflux surgery: a study of prevalence and predictive features. J. Am. Coll. Surg. 196: 706712.

40. Csendes, A., I. Braghetto, et al. 2009. Late results of surgical treatments of 125 patients with short-segment Barrett's esophagus. Arch. Surg. 144: 921-927.

41. Hofstetter, W.L., J.H. Peters, et al. 2001. Long-term outcome of anti-reflux surgery in patients with Barrett's esophagus. Ann. Surg. 234: 532-539.

42. Parrilla, P., L.F. Martinez de Haro, et al. 2003. Long-term results of a randomized prospective study comparing medi- cal and surgical treatment of Barrett's esophagus. Ann. Surg. 237: 291-298.

43. Rossi, M., M. Barreca, et al. 2006. Efficacy of Nissen fundoplication versus medical therapy in the regression of low-grade dysplasia in patients with Barrett esophagus. A prospective study. Ann. Surg. 243: 58-63.

44. Martinez de Haro, L., A. Ortiz, et al. 2001. Intestinal metaplasia in patients with columnar lined esophagus is associated with high levels of duodenogastroesphageal reflux. Ann. Surg. 233: 34-38.

45. Todd, J.A., K.K. Basu, et al. 2005. Normalisation of oesophageal $\mathrm{pH}$ does not guatantee control of duodenogastroesophageal reflux in Barrett's esophagus. Aliment. Pharmacol. Ther. 21: 969-975.

46. Chang, E.Y., C.D. Morris, et al. 2007. The effect of antireflux surgery on esophageal carcinogenesis in patients with Barrett's esophagus. Ann. Surg. 246: 11-21.

47. Corey, K.E., S.M. Schmitz, et al. 2003. Does a surgical antireflux procedure decrease the incidence of esophageal adenocarcinoma in Barrett's esophagus? A meta-analysis. Am. J. Gastroenterol. 98: 2390-2394.

48. Lambert, R. \& P. Hainaut. 2007. Esophageal cancer: the precursors (Part II). Endoscopy 39: 659-664.

49. Singh, R., G.K. Anagnostopoulos, K. Yao, et al. 2008. Narrow-band imaging with magnification in Barrett's esophagus: validation of a simplified grading system of mucosal morphology patterns against histology. Endoscopy 40: 457-463.

50. Mannath, J., V. Subramanian, C.J. Hawkey, et al. 2010. Narrow band imaging for characterization of high-grade dysplasia and specialized intestinal metaplasia in Barrett's esophagus: a meta-analysis. Endoscopy 42: 351-359.

51. Kara, M.A., F.P. Peters, P. Fockens, et al. 2006. Endoscopic video-autofluorescence imaging followed by narrow band imaging for detecting early neoplasia in Barrett's esophagus. Gastrointest. Endosc. 64: 176-185.

52. Curvers, W.L., L.A. Herrero, M.B. Wallace, et al. 2010. Endoscopic tri-modal imaging is more effective than standard endoscopy in targeting early-stage neoplasia in Barrett's esophagus. Gastroenterology [Epub ahead of print].

53. Maru, D.M. 2009. Barrett's esophagus: diagnostic challenges and recent developments. Ann. Diag. Path. 13: 212-221.

54. Reid, B.J., D.S. Levine, G. Longton, et al. 2000. Predictors of progression to cancer in Barrett's esophagus: baseline histology and flow cytometry identify low- and high-risk patient subsets. Am. J. Gastroenterol. 95: 1669-1676.

55. Schnell, T.G., S.J. Sontag, G. Chejfec, et al. 2001. Long-term nonsurgical management of Barrett's esophagus with highgrade dysplasia. Gastroenterology 120: 1607-1619.

56. Ell, C., A. May, L. Grossner, et al. 2000. Endoscopic mucosal resection for early cancer and high-grade dysplasia in Barrett's esophagus. Gastroenterology 118: 670-677.

57. Shaheen, N.J., P. Sharma, B.F. Overholt, et al. 2009. Radiofrequency ablation in Barrett's esophagus with dysplasia. N. Engl. J. Med. 360: 2277-2288. 